\title{
Heart defects-limb shortening syndrome
}

INSERM

\section{Source}

INSERM. (1999). Orphanet: an online rare disease and orphan drug data base. Heart defects-limb shortening syndrome. ORPHA:1354

A rare multiple congenital anomalies/dysmorphic syndrome characterized by skeletal dysplasia (including coronal clefting of the vertebral bodies and short limbs) and variable congenital heart malformations, such as atrial and ventricular septal defects, right ventricular hypoplasia, and valve defects). There have been no further descriptions in the literature since 1990. 